\title{
Moduli portal dark matter
}

\author{
Debtosh Chowdhury, ${ }^{1,2, *}$ Emilian Dudas, ${ }^{1, \dagger}$ Maíra Dutra, ${ }^{2,3, \$}$ and Yann Mambrini ${ }^{2, \S}$ \\ ${ }^{1}$ Centre de Physique Théorique, École Polytechnique, CNRS, Université Paris-Saclay, \\ 91128 Palaiseau Cedex, France \\ ${ }^{2}$ Laboratoire de Physique Théorique (UMR8627), CNRS, Univ. Paris-Sud, \\ Université Paris-Saclay, 91405 Orsay, France \\ ${ }^{3}$ Ottawa-Carleton Institute for Physics, Carleton University, \\ 1125 Colonel By Drive, Ottawa, K1S 5 B6 Ontario, Canada
}

(Received 19 November 2018; published 23 May 2019)

\begin{abstract}
We show that moduli fields as mediators between the Standard Model and the dark sector can naturally lead to the observed relic abundance. Indeed, even if moduli are very massive, the nature of their couplings with matter and gauge fields allows to produce a sufficiently large amount of dark matter in the early Universe through the freeze-in mechanism. Moreover, the complex nature of the moduli fields whose real and imaginary parts couple differently to the thermal bath gives an interesting and unusual phenomenology compared to other freeze-in models of that type.
\end{abstract}

DOI: $10.1103 /$ PhysRevD.99.095028

\section{INTRODUCTION}

Despite indirect but clear evidence [1] of the presence of a large amount of dark matter (DM) in our Universe, its nature still remains elusive. The absence of any signal in direct-detection experiments like XENON [2], LUX [3], and PANDAX [4] questions the weakly coupled dark matter paradigm. The simplest extensions-such as Higgs-portal [5], Z-portal [6], or even $Z^{\prime}$-portal models [7] - have a large part (if not all) of their parameter space excluded when combining direct, indirect, and accelerator searches (for a review on weakly interacting massive particle searches and models, see Ref. [8]). This uncomfortable situation justifies the need to look for different scenarios, allowing feeble couplings, or the possibility of dark matter production at the very early stages of reheating. Such mechanisms are usually dubbed "feebly interacting massive particles" [9] (see Ref. [10] for a review).

In this context, several models have been studied and it has been confirmed that dark matter production is naturally feasible in different setups like $\mathrm{SO}(10)$ unified construction [11], $\mathrm{U}(1)^{\prime}$ anomaly-free models [12], the spin-2 portal [13], or high-scale supersymmetry (SUSY) [14]. In all off

\footnotetext{
*debtosh.chowdhury@polytechnique.edu

†emilian.dudas@polytechnique.edu

*maira.dutra@th.u-psud.fr; mdutra@physics.carleton.ca

§yann.mambrini@th.u-psud.fr
}

Published by the American Physical Society under the terms of the Creative Commons Attribution 4.0 International license. Further distribution of this work must maintain attribution to the author(s) and the published article's title, journal citation, and DOI. Funded by SCOAP ${ }^{3}$. these models, it has been shown that the effects of noninstantaneous reheating [15] and noninstantaneous thermalization [16] should be considered with care. It then seemed interesting to study constructions with massive scalar moduli fields which are abundantly present in supergravity (SUGRA) and string theory extensions, and to check if they can play the role of a mediator between the dark sector and the Standard Model (SM). Indeed, moduli fields couple generically to Standard Model fields through higher-dimensional operators (mostly with derivative interactions). As a consequence, mechanisms implying moduli are important at high energies/temperatures, being therefore potentially relevant for the freeze-in mechanism.

Moduli fields appear in many extensions of the Standard Model. Indeed, in any higher-dimensional supergravity or string theory extension of the Standard Model there are scalar fields coming from the compactification of the higher-dimensional metric, dilaton, or various antisymmetric tensors. In particular, internal volumes and shapes and their axionic partners are abundant in such constructions. Most of them are flat directions at tree level and get potentials and therefore masses from various perturbative and nonperturbative effects. Their resulting masses and vacuum expectation values (VEVs) are model dependent and will be taken as free parameters in what follows. Their VEVs determine the values of four-dimensional parameters: the gauge and Yukawa couplings, the wave functions of various fields, and the Planck mass. If one assumes that the low-energy theory (obtained after their decoupling) is the Standard Model or a phenomenologically motivated extension of it, then their couplings can be obtained by 
starting from the low-energy theory and expanding the lowenergy parameters in a power series. If moduli fields are heavier than the reheating temperature, then they can be safely replaced by their VEVs and the low-energy theory is just the Standard Model or an appropriate extension. If they are lighter, however, they can lead to various physical effects. This strategy was used in early papers [17-23] in order to study various low-energy effects of the moduli fields. Recent works have considered moduli as dark matter candidates [24] or the production of dark matter from moduli decay in the early Universe [25-29]. The present work is intended to complete the list of interesting consequences of moduli fields by studying their possible role as mediators between the Standard Model and the dark matter sector.

The paper is organized as follows. In Sec. II, we describe the model under consideration. In Sec. III, we discuss bounds on the moduli masses coming from cosmology. In Sec. IV, we outline the dark matter relic abundance through the freeze-in mechanism in the early Universe, taking into account noninstantaneous reheating. Section V is devoted to the computation of the dark matter production rate in the early Universe in our model and we delineate the parameter space for the model in consideration. In Sec. VI, we summarize the main results of our work and conclude by highlighting the new aspects that emerged from our analysis.

\section{THE MODEL}

Let $\Lambda$ be the new physics scale (which can be the string scale, unification scale, or SUSY/SUGRA breaking scale, for instance). The consistency of the effective field theory requires that $\Lambda$ is the largest mass scale of the theory-in particular, larger than the dark matter or mediator masses and the maximum temperature after reheating. One can then define the couplings of the complex modulus field ${ }^{1} \mathcal{T}$ (decomposed as $\mathcal{T} \equiv t+i a$ ) to the Standard Model field $k$ by expanding the wave functions $\mathcal{Z}_{k}$ :

$$
\mathcal{Z}_{k}(\mathcal{T}, \overline{\mathcal{T}}) \approx 1+\frac{c_{k}}{\Lambda} \mathcal{T}+\frac{d_{k}}{\Lambda} \overline{\mathcal{T}} \equiv 1+\frac{\alpha_{k}}{\Lambda} t+i \frac{\beta_{k}}{\Lambda} a
$$

where $c_{k}$ and $d_{k}$ are real coefficients of order one and we defined the couplings to the real and imaginary components of $\mathcal{T}$ as $\alpha_{k}=c_{k}+d_{k}$ and $\beta_{k}=c_{k}-d_{k}$, respectively. ${ }^{2} \mathrm{We}$ can then express generic couplings of the moduli fields to the Standard Model sector as

\footnotetext{
${ }^{1}$ We will consider only one modulus field throughout our work. The generalization to several fields is straightforward.

${ }^{2}$ For simplicity, throughout our work we will consider $C P$ conserving Lagrangians and therefore real coefficients in the couplings of moduli to matter. The extension to $C P$-violating couplings is interesting but beyond the goal of our paper.
}

$$
\begin{aligned}
\mathcal{L}_{\mathcal{T}}^{\mathrm{SM}} \supset & \mathcal{Z}_{H}\left|D_{\mu} H\right|^{2}-\mu^{2}(\mathcal{T}, \overline{\mathcal{T}})|H|^{2}-\lambda(\mathcal{T}, \overline{\mathcal{T}})|H|^{4} \\
& +\frac{1}{2}\left(\mathcal{Z}_{L} \bar{f}_{L} i \not D f_{L}+\mathcal{Z}_{R} \bar{f}_{R} i \not D f_{R}+\text { H.c. }\right) \\
& -\frac{1}{4} \mathcal{Z}_{G} G_{\mu \nu} G^{\mu \nu}-\mathcal{Z}_{G}^{\prime} G_{\mu \nu} \tilde{G}^{\mu \nu},
\end{aligned}
$$

where $\mathcal{Z}_{H}\left(=1+\frac{\alpha_{H}}{\Lambda} t\right), \quad \mathcal{Z}_{L, R}\left(=1+\frac{\alpha_{L, R}}{\Lambda} t+i \frac{\beta_{L, R}}{\Lambda} a\right)$, $\mathcal{Z}_{G}\left(=1+\frac{\alpha_{G}}{\Lambda} t\right)$, and $\mathcal{Z}_{G}^{\prime}\left(=\frac{\beta_{G}}{\Lambda} a\right)$ are the wave functions of the scalar $(H)$, fermionic $(f)$, and (Abelian and nonAbelian) gauge fields $\left(G_{\mu}\right)$ of the Standard Model, respectively. In the above equation, $G_{\mu \nu}$ is the field-strength tensor of the gauge field $\left(G_{\mu}\right)$ and $\tilde{G}^{\mu \nu}\left(=\frac{1}{2} \epsilon^{\mu \nu \rho \sigma} G_{\rho \sigma}\right)$ is its dual field-strength tensor.

From the first line of Eq. (2) we see that the scalar potential depends on the mass parameter $\mu$, which is also a function of the moduli fields. Parametrizing the contribution of the moduli to the $\mu$ parameter in a similar fashion as in Eq. (1), we can write

$$
\mu^{2}=\mu_{0}^{2}\left(1+\frac{\alpha_{\mu}}{\Lambda} t\right)
$$

with $\mu_{0}$ being the SM $\mu$ parameter that reproduces the observed Higgs mass at the electroweak scale. As $\Lambda$ is the highest scale in the theory, the contribution to the Higgs mass due to the moduli is small. On the other hand, there is a second possibility: the $\mu$ parameter gets generated at a scale $(\sqrt{\langle F\rangle})$ close to the Planck scale. In this case, the effective $\mu$ parameter can be written as

$$
\mu^{2}=\mu_{0}^{2}+\frac{\langle F\rangle}{M_{P}} t
$$

where $\langle F\rangle$ is the VEV of the "spurion" field. In this case, one needs a considerable amount of cancellation or fine-tuning between the two contributions in Eq. (4) to reproduce the observed Higgs mass. In contrast to Eq. (3), in this case the coupling of $t$ to the Higgs is quite large. This leads to the fact that the width of $t$ could be larger than the mass of $t$ unless we demand that the width be at most the mass of $t$. This sets an upper bound on the spurion VEV, $\langle F\rangle \lesssim m_{t} M_{P}$, where $m_{t}$ is the mass of $t$. Throughout our analysis, we will consider the case of Eq. (3) unless otherwise stated.

The effective interactions between the components of the moduli and SM fields, at the first order in $1 / \Lambda$, reads

$$
\begin{aligned}
\mathcal{L}_{\mathcal{T}}^{\mathrm{SM}} \supset & \frac{\alpha_{H}}{\Lambda} t\left|D_{\mu} H\right|^{2}-\frac{\alpha_{\mu}}{\Lambda} \mu_{0}^{2} t|H|^{2} \\
& +\left(\frac{1}{2 \Lambda} t \bar{f} i \gamma^{\mu}\left(\alpha_{\mathrm{V}}^{f}-\alpha_{\mathrm{A}}^{f} \gamma_{5}\right) D_{\mu} f+\text { H.c. }\right) \\
& +\frac{1}{2 \Lambda} \partial_{\mu} a \bar{f} \gamma^{\mu}\left(\beta_{\mathrm{V}}^{f}-\beta_{\mathrm{A}}^{f} \gamma_{5}\right) f \\
& -\frac{1}{4} \frac{\alpha_{G}}{\Lambda} t G_{\mu \nu} G^{\mu \nu}+2 \frac{\beta_{G}}{\Lambda} \partial_{\mu} a \epsilon^{\mu \nu \rho \sigma} G_{\nu} \partial_{\rho} G_{\sigma},
\end{aligned}
$$


where we have identified the chiral couplings as $\alpha_{\mathrm{V}}^{f}=\left(\alpha_{L}+\alpha_{R}\right) / 2$ and $\alpha_{\mathrm{A}}^{f}=\left(\alpha_{L}-\alpha_{R}\right) / 2$, with analogous definitions for the couplings of the imaginary part of the moduli.

Before we proceed further we can make some remarks after having a quick look at Eq. (5):

(1) Since the kinetic term of the Higgs needs to be real, the Higgs sector only couples with the real part of the modulus field $t$.

(2) One observes that the Lagrangian in Eq. (5) is invariant under a shift in the imaginary part of the moduli ( $a \rightarrow a+$ const). This can also be observed in SUGRA models, for instance, where the Kähler metric depends explicitly on the combination $\mathcal{T}+\overline{\mathcal{T}}$. In other words, the Lagrangian could be written by imposing the shift symmetry from the beginning. ${ }^{3}$ As a consequence, the nature of the couplings of $a$ to the Standard Model fields differs from the couplings of $t$ ( $a$ naturally develops derivative-type couplings).

(3) In Eq. (5) we kept only the leading operators which contribute to three-point functions. This is because the $2 \rightarrow 2$ production of dark matter will dominate over the $3 \rightarrow 2$ production. ${ }^{4}$

By analogy, one can write the same type of couplings in the dark sector. In our study, we will distinguish three cases of dark mater: scalar $(S)$, fermionic $(\chi)$, and vectorial $(\mathcal{V})$. Their interactions with moduli read

$$
\begin{gathered}
\mathcal{L}_{\mathcal{T}}^{S}=\frac{\alpha_{S}}{\Lambda} t\left|\partial_{\mu} S\right|^{2}, \\
\mathcal{L}_{\mathcal{T}}^{\chi}=\left(\frac{1}{2 \Lambda} t \bar{\chi} i \gamma^{\mu}\left(\alpha_{\mathrm{V}}^{\chi}-\alpha_{\mathrm{A}}^{\chi} \gamma_{5}\right) \partial_{\mu} \chi+\text { H.c. }\right) \\
+\frac{1}{2 \Lambda} \partial_{\mu} a \bar{\chi} \gamma^{\mu}\left(\beta_{\mathrm{V}}^{\chi}-\beta_{\mathrm{A}}^{\chi} \gamma_{5}\right) \chi, \\
\mathcal{L}_{\mathcal{T}}^{\mathcal{V}}=-\frac{1}{4} \frac{\alpha_{\mathcal{V}}}{\Lambda} t \mathcal{V}_{\mu \nu} \mathcal{V}^{\mu \nu}+2 \frac{\beta_{\mathcal{V}}}{\Lambda} \partial_{\mu} a \epsilon^{\mu \nu \rho \sigma} \mathcal{V}_{\nu} \partial_{\rho} \mathcal{V}_{\sigma} .
\end{gathered}
$$

We can make some remarks concerning the couplings of the moduli fields to the dark matter. First of all, similar to the case for the Higgs sector, the scalar dark matter does not couple to the imaginary part of the moduli. An immediate consequence (which will be discussed in what follows) is that only the real part of moduli contribute to the production of a scalar dark matter, regardless of the Standard Model initial states.

\footnotetext{
${ }^{3}$ This argument is not valid if one includes Yukawa-like couplings in the action. However, their contribution to dark matter production in the early Universe is negligible.

${ }^{4} 3 \rightarrow 2$ processes can dominate if $2 \rightarrow 2$ channels are suppressed, and when strong interactions are generated to compensate the loss induced by the reduced phase space [30].
}

The interactions of fermions ( $\Psi$, standard or dark) with moduli are essentially different from the scalar and vectorial cases, due to their chirality. The first aspect of this remark is evident from the amplitudes for their interactions with moduli:

$$
\begin{aligned}
& \mathcal{M}_{t \bar{\Psi} \Psi}=-\frac{i}{2 \Lambda} \bar{u}\left(p_{1}\right)\left(\not p_{1}-\not p_{2}\right)\left(\alpha_{\mathrm{V}}^{\Psi}-\alpha_{\mathrm{A}}^{\Psi} \gamma_{5}\right) v\left(p_{2}\right) \\
&=-i \frac{\alpha_{\mathrm{V}}^{\Psi} m_{\Psi}}{\Lambda} \bar{u}\left(p_{1}\right) v\left(p_{2}\right), \\
& \mathcal{M}_{a \bar{\Psi} \Psi}=\frac{1}{2 \Lambda} \bar{u}\left(p_{1}\right)(\not \not p 1 \\
&\left.1 \not p_{2}\right)\left(\beta_{\mathrm{V}}^{\Psi}-\beta_{\mathrm{A}}^{\Psi} \gamma_{5}\right) v\left(p_{2}\right) \\
&=-\frac{\beta_{\mathrm{A}}^{\Psi} m_{\Psi}}{\Lambda} \bar{u}\left(p_{1}\right) \gamma_{5} v\left(p_{2}\right),
\end{aligned}
$$

where $p_{1}$ and $p_{2}$ are the four-momenta of the fermions and $m_{\Psi}$ is the mass of the fermion. We notice that if the fermions are on shell, we have an explicit dependence on their mass due to the Dirac equation. As a consequence, above the electroweak scale, standard fermions cannot produce any of the dark matter particles. The other aspect we point out is that the fermionic coupling to the real part of the moduli is $C P$ even, so that the corresponding rates will depend only on the vector coupling $\left(\alpha_{\mathrm{V}}^{\Psi}\right)$, and that the fermionic coupling to the imaginary part of the moduli is $C P$ odd and therefore the corresponding rates will depend only on the axionic coupling $\left(\beta_{\mathrm{A}}^{\Psi}\right)$.

\section{COSMOLOGICAL MODULI PROBLEM}

Because of their gravitational interactions, moduli are long-lived fields. Thus, one has to face two potential dangers. If the moduli lifetime is smaller than the age of our Universe, their decay might have released quite a large amount of entropy in the Universe which would dilute the contents of the Universe. On the other hand, if the moduli lifetime is larger than the age of our Universe, they might still be oscillating around the minimum of their potential. Thus, the energy stored in these oscillations may overclose the Universe. One refers to these problems as the cosmological moduli problem [31]. Taken at their face values, such constraints dictate that the moduli fields are either superlight or superheavy. We present here a short review on these well-known constraints, following Ref. [31].

Using simple dimensional analysis the decay width of a modulus field $\mathcal{T}$ of mass $m_{\mathcal{T}}$ can be written as $\Gamma_{\mathcal{T}} \sim m_{\mathcal{T}}^{3} / M_{P}^{2}$. Since the age of the Universe is of order $H_{0}^{-1}$, where $H_{0}$ is the Hubble constant in the present Universe, we could infer that the modulus will decay at present times if $\Gamma_{\mathcal{T}} \sim H_{0}$, or in other words if its mass $m_{\mathcal{T}}$ is around $\left(H_{0} M_{P}^{2}\right)^{1 / 3} \simeq 20 \mathrm{MeV}$.

At first, we consider the case when $m_{\mathcal{T}}<20 \mathrm{MeV}$, that is, when the modulus has not decayed yet at the present time. As long as the Hubble constant is larger than the modulus mass, the friction term $(3 H \dot{\mathcal{T}})$ dominates in the 
equation of motion of the modulus field and the field $\mathcal{T}$ remains frozen at its initial value $f_{\mathcal{T}}$. When the Hubble constant is of the order of the mass of the modulus or $H \sim m_{\mathcal{T}}$, that is, when $T_{I} \sim \sqrt{m_{\mathcal{T}} M_{P}}$ (since we know that $\left.H \sim T^{2} / M_{P}\right)$, the field $\mathcal{T}$ starts oscillating around the minimum $\mathcal{T}_{0}$ of its potential. These coherent oscillations behave like nonrelativistic matter, and thus the energy density of the modulus reads

$$
\rho_{\mathcal{T}}(T)=\rho_{\mathcal{T}}\left(T_{I}\right)\left(\frac{T}{T_{I}}\right)^{3} \sim m_{\mathcal{T}}^{2} f_{\mathcal{T}}^{2}\left(\frac{T}{T_{I}}\right)^{3} .
$$

We know that the radiation energy density $\rho_{\gamma}(T)$ scales as $T^{4}$, and thus $\rho_{\mathcal{T}} / \rho_{\gamma}$ scales as $1 / T$. As the temperature of the Universe decreases the energy fraction stored in the moduli increases. Then, there will be a time when the energy density of the modulus oscillations will dominate the total energy density of the Universe. Thus, one needs to make sure that in the present Universe $\rho_{\mathcal{T}}\left(T_{0}\right)<\rho_{c}$, where $T_{0}$ is the temperature of the present Universe and $\rho_{c}$ is the critical density. Using the above equation and $T_{I}=\sqrt{m_{\mathcal{T}} M_{P}}$, one can write this condition as

$$
m_{\mathcal{T}}<M_{P}\left(\frac{\rho_{c} M_{P}}{f_{\mathcal{T}}^{2} T_{0}^{3}}\right)^{2} \sim 10^{-26} \mathrm{eV},
$$

where we have assumed $f_{\mathcal{T}} \simeq M_{P}$. Thus, if $10^{-26} \mathrm{eV}<$ $m_{\mathcal{T}}<20 \mathrm{MeV}$, there is a huge amount of energy stored in the $\mathcal{T}$ field.

Next, we consider the case when $m_{\mathcal{T}}>20 \mathrm{MeV}$, that is, when the modulus field has already decayed at the present time. Assuming the modulus field energy density dominates over the radiation energy density, the decay of the modulus occurs at a temperature $T_{D}$ when $H\left(T_{D}\right) \sim \Gamma_{\mathcal{T}}$, which can be reexpressed as

$$
\Gamma_{\mathcal{T}}^{2} \sim \frac{\rho_{\mathcal{T}}\left(T_{D}\right)}{M_{P}^{2}} .
$$

At the time of decay, all of the energy density stored in the modulus is transferred into radiation energy density. Thus, the reheating temperature $\left(T_{\mathrm{RH}}\right)$ is given by the condition $\rho_{\mathcal{T}}\left(T_{D}\right) \sim T_{\mathrm{RH}}^{4}$. Using the above equation, we can obtain

$$
T_{\mathrm{RH}} \sim \sqrt{M_{P} \Gamma_{\mathcal{T}}} \simeq \sqrt{\frac{m_{\mathcal{T}}^{3}}{M_{P}}} .
$$

The entropy release due to the modulus decay must take place before big bang nucleosynthesis such that the abundance of the light elements is not affected. This condition, namely, $T_{\mathrm{RH}}>1 \mathrm{MeV}$ [32], gives $m_{\mathcal{T}}>10 \mathrm{TeV}$. Thus, for $20 \mathrm{MeV}<m_{\mathcal{T}}<10 \mathrm{TeV}$, the entropy release due to the decay of the modulus field would contradict current cosmological observations. In the absence of any other effects, a cosmologically acceptable regime for the modulus mass is either superlight $\left(m_{\mathcal{T}}<10^{-26} \mathrm{eV}\right)$ or superheavy $\left(m_{\mathcal{T}}>10 \mathrm{TeV}\right)$. This concludes the short review discussing the constraints on the moduli mass.

\section{DARK MATTER PRODUCTION}

The evolution of the dark matter number density $n_{\mathrm{DM}}$ is determined by the Boltzmann equation

$$
\frac{\mathrm{d} n_{\mathrm{DM}}}{\mathrm{d} t}=-3 H(t) n_{\mathrm{DM}}+R(T),
$$

where $H(t)=\frac{1}{\sqrt{3} M_{P}} \sqrt{\rho_{\text {tot }}(t)}$ is the Hubble expansion rate, with $M_{P} \simeq 2.4 \times 10^{18} \mathrm{GeV}$ being the reduced Planck mass and $\rho_{\text {tot }}(t)$ the total energy density which changes with time, as we will see in what follows. $R(T)=$ $n_{\mathrm{SM}}^{2}\langle\sigma v\rangle_{\mathrm{SMSM} \rightarrow \mathrm{DMDM}}$ is the production rate (the number of dark matter particles produced per unit of time and unit of volume; see the Appendix B for details) which, for a process $(1,2 \rightarrow 3,4)$, is given by ${ }^{5}$

$R(T)=\int f_{1} f_{2} \frac{E_{1} E_{2} \mathrm{~d} E_{1} \mathrm{~d} E_{2} \mathrm{~d} \cos \theta_{12}}{1024 \pi^{6}} \int|\mathcal{M}|_{i}^{2} \mathrm{~d} \Omega_{13}$,

where $E_{i}$ and $f_{i}$ are the energy and (thermal) distribution function of particle $i$, respectively, $\theta_{12}$ is the angle between the incoming particles 1 and 2 in the laboratory frame, and $\Omega_{13}$ is the solid angle between the outgoing particles 1 and 3 in the rest frame.

Since we are interested in mediators with masses that could be near the reheating scale, the contribution to the total energy density of the inflaton field (labeled by $\phi$ ) may dominate over the contribution of radiation (labeled by $\gamma$ ) in the Hubble rate. In order to find the correct amount of dark matter, Eq. (14) needs to be solved numerically along with the following coupled equations (see, for instance, Refs. [15,33]):

$$
\begin{aligned}
& \frac{\mathrm{d} \rho_{\gamma}}{\mathrm{d} t} \approx-4 H \rho_{\gamma}+\Gamma_{\phi} \rho_{\phi}, \\
& \frac{\mathrm{d} \rho_{\phi}}{\mathrm{d} t}=-3 H \rho_{\phi}-\Gamma_{\phi} \rho_{\phi},
\end{aligned}
$$

where the approximation neglects the effect of interactions with dark matter particles in the evolution of the energy density of radiation, which is dominated by the decay of the inflaton field, with a total decay width $\Gamma_{\phi}$. We have defined

\footnotetext{
${ }^{5}$ In our analysis we assume the initial value of the modulus field to be around $10^{10} \mathrm{GeV}$ such that DM production from the modulus decay remains subdominant with respect to the $\mathrm{SMSM} \rightarrow$ DMDM process.
} 
$T_{\mathrm{RH}}$ as the temperature in a radiation-dominated Universe after the inflaton decay, $\Gamma_{\phi}=H\left(T_{\mathrm{RH}}\right)$.

In the present work, we have solved the set of three coupled differential equations above, but it is instructive to find analytic solutions for the limiting cases of inflaton and radiation domination. In fact, we have checked that this is a good approximation, since there is a recognizable change of regime in the Hubble rate near the reheating temperature (see, for instance, Ref. [34]). In the radiation-dominated era, we can use the familiar relations ${ }^{6}$

$$
\frac{\mathrm{d}}{\mathrm{d} t}=-H(T) T \frac{\mathrm{d}}{\mathrm{d} T} \quad \text { with } \quad H(T)=\sqrt{\frac{g_{e}}{90}} \pi \frac{T^{2}}{M_{P}},
$$

while in the inflaton-dominated era, we have [12]

$$
\frac{\mathrm{d}}{\mathrm{d} t}=-\frac{3}{8} H(T) T \frac{\mathrm{d}}{\mathrm{d} T} \text { with } H(T)=\sqrt{\frac{5 g_{\mathrm{MAX}}^{2}}{72 g_{\mathrm{RH}}}} \pi \frac{T^{4}}{T_{\mathrm{RH}}^{2} M_{P}},
$$

where $g_{\mathrm{RH}}$ and $g_{\mathrm{MAX}}$ are, respectively, the relativistic degrees of freedom at the reheating temperature after inflation and at the maximal temperature reached during the reheating process, $T_{\mathrm{MAX}}$.

The dark matter relic density $\Omega h^{2} \equiv m_{\mathrm{DM}} n_{\mathrm{DM}} / \rho_{c}$, where $\rho_{c}$ is the critical density today, may be split into radiation-dominated (RD) and inflaton-dominated (ID) contributions:

$$
\begin{aligned}
\Omega h^{2} \cong & \Omega h_{\mathrm{RD}}^{2}+\Omega h_{\mathrm{ID}}^{2} \\
& \sim 4 \times 10^{24} m_{\mathrm{DM}}\left(\int_{T_{0}}^{T_{\mathrm{RH}}} d T \frac{R(T)}{T^{6}}\right. \\
& \left.+1.07 T_{\mathrm{RH}}^{7} \int_{T_{\mathrm{RH}}}^{T_{\mathrm{MAX}}} d T \frac{R(T)}{T^{13}}\right) \\
& \equiv h_{\mathrm{RD}}^{2} B_{F},
\end{aligned}
$$

where $T_{0}$ is the temperature of the present Universe. In the above equation we have defined a "boost factor" $B_{F}$ $\left(=1+\frac{\Omega h_{\mathrm{ID}}^{2}}{\Omega h_{\mathrm{RD}}^{2}}\right)$, which quantifies the fraction of dark matter produced during the ID era to the amount of dark matter produced during the RD era. Notice that the fraction of dark matter produced during the reheating stage is $\left(B_{F}-1\right) / B_{F}$.

From this expression we can see that the production of dark matter during reheating $\left(T_{\mathrm{RH}}<T<T_{\mathrm{MAX}}\right)$ might be relevant if the temperature dependence in the rate is sufficiently high. Let us parametrize the rate as $R(T) \propto$ $T^{n}$ and denote the boost factor as $B_{F}^{(n)}$. For $n \geq 12$, dark matter production during reheating is comparable to or

\footnotetext{
${ }^{6}$ For simplicity, we will not assume changes in the energetic and entropic relativistic degrees of freedom, $g_{e}$ and $g_{s}$, respectively. In what follows, we set $g_{e}=g_{s}=106.75$.
}

dominant over production during the radiation-dominated era. For instance, for $T_{\mathrm{MAX}}=100 T_{\mathrm{RH}}$ we find

$$
\begin{gathered}
B_{F}^{(10)} \approx 1+1.07 \times \frac{5}{2}\left(1+\frac{T_{\mathrm{RH}}^{2}}{T_{\mathrm{MAX}}^{2}}\right) \simeq 3.68, \\
B_{F}^{(12)} \approx 1+1.07 \times 7 \ln \left(\frac{T_{\mathrm{MAX}}}{T_{\mathrm{RH}}}\right) \simeq 35.5,
\end{gathered}
$$

and for $n>12$,

$$
B_{F}^{(n>12)} \approx 1+1.07 \times \frac{n-5}{n-12}\left(\frac{T_{\mathrm{MAX}}}{T_{\mathrm{RH}}}\right)^{n-12} .
$$

The percentage of dark matter production during reheating for $n=10$ is therefore $\sim 73 \%$, whereas for $n=$ 12 it is $\sim 97 \%$. An important point we want to emphasize here is that if the mediators between the dark and visible sectors are close to the reheating scale and if the production rate of dark matter has a high temperature dependence, we cannot avoid the contribution of the inflaton to the Hubble rate.

\section{RESULTS AND DISCUSSION}

\section{A. Production rate}

The squared amplitudes responsible for the modeldependent behavior of the production rates of three different dark matter candidates are given in Appendix B. ${ }^{7}$ Before presenting the exact solution of the rate [see Eq. (B4)], we recognize from the squared amplitudes three regimes which depend on the relation between the mass of the mediators and the temperature of the thermal bath:

(1) The light regime, when the mediator mass is much below the temperature of the thermal bath $T\left(m_{t, a} \ll T\right)$.

(2) The pole regime, when the mediator mass is of the same order as the temperature $\left(m_{t, a} \sim T\right)$, where we might use the narrow-width approximation (NWA).

(3) The heavy regime, when the mediator mass is much above the temperature $\left(m_{t, a} \gg T\right)$.

Far from the pole of the propagator, we might assume $\Gamma_{t, a} \ll m_{t, a}$. In the limit $m_{\mathrm{DM}} \ll T$, we can obtain analytic solutions for the production rate $R_{s_{f}}^{j}$ of dark matter of spin $s_{f}$ due to the exchange of a mediator $j$ :

\footnotetext{
${ }^{7}$ In our analysis, we concentrate on the first possibility discussed for the mass parameter of the Higgs [see Eq. (3)]. For the possibility where the mass parameter is given by Eq. (4), the result is similar to the case in Eq. (3).
} 


$$
\begin{aligned}
& R_{0,1}^{j}(T)=\delta_{0,1}^{j} \times \begin{cases}\frac{T^{8}}{\Lambda^{4}} & \left(m_{j} \ll T\right), \\
\frac{m_{j}^{8}}{\Lambda^{4}} \frac{T}{\Gamma_{j}} K_{1}\left(\frac{m_{j}}{T}\right) & \left(m_{j} \sim T\right), \\
\frac{T^{12}}{m_{j}^{4} \Lambda^{4}} & \left(m_{j} \gg T\right),\end{cases} \\
& R_{1 / 2}^{j}(T)=\delta_{1 / 2}^{j} \begin{cases}\frac{m_{\mathrm{DM}}^{2} T^{6}}{\Lambda^{4}} & \left(m_{j} \ll T\right), \\
\frac{m_{\mathrm{DM}}^{2} m_{j}^{6}}{\Lambda^{4}} \frac{T}{\Gamma_{j}} K_{1}\left(\frac{m_{j}}{T}\right) & \left(m_{j} \sim T\right), \\
\frac{m_{\mathrm{DM}}^{2} T^{10}}{m_{j}^{4} \Lambda^{4}} & \left(m_{j} \gg T\right),\end{cases}
\end{aligned}
$$

where the proportionality constants $\delta_{s_{f}}^{j}$ are given in Table II.

We have numerically computed the total production rates (B4), where the integration was performed using the CUBA package [35], with a Bose-Einstein distribution function for the Higgs and gauge bosons in the initial states.

In Fig. 1, we show the exact solutions of the total production rate of the fermionic dark matter for a representative set of free parameters, as a function of the variable $x=m_{s} / T$ which may be regarded as a parametrization of time. We set the new physics scale $\Lambda$ to be $10^{16} \mathrm{GeV}$ (the grand unified theory scale), $T_{\mathrm{MAX}}=10^{12} \mathrm{GeV}$, and the mass of the axionic modulus to be $10^{8} \mathrm{GeV}$. For simplicity, all of the couplings are set to unity. From left to right, the mass of the real component of the modulus is set to $10^{10}$, $10^{13}$, and $10^{15} \mathrm{GeV}$ (green, orange, and blue curves, respectively). The mass of the fermionic dark matter is set to be between the mediator masses in the first case $\left(10^{9} \mathrm{GeV}\right)$ and to be relatively light in the second and third cases $\left(10^{4} \mathrm{GeV}\right)$.

It is easy to understand the mechanism at work in the dark matter production after taking a look at Fig. 1. First of all, a general feature of the rate is the strong temperature dependence: the higher the temperature (small- $x$ region), the more dark matter will be produced. The second generic feature is the threshold for dark matter production, which is due to the Boltzmann-suppressed photon distribution having $T<m_{\mathrm{DM}}$ (large $x$ ). This happens just after $x=10,10^{9}$, and $10^{11}$ for the three case, respectively.

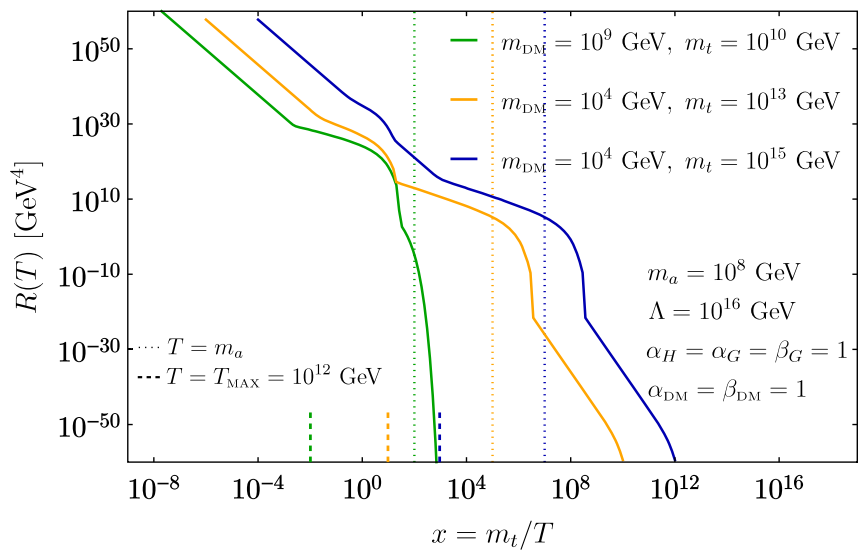

FIG. 1. Evolution of the production rate of fermionic dark matter as a function of the temperature for different masses of dark matter and a real component of the modulus field.

Between those two extremes, we can notice the effects of the pole regions once $T$ reaches $m_{t}(x \sim 1)$ and $m_{a}\left(x=10^{2}\right.$, $10^{5}$, and $10^{7}$ for $m_{t}=10^{10}, 10^{13}$, and $10^{15} \mathrm{GeV}$, respectively). Notice that the production rates for the scalar dark matter would not have the effect of the poles of $m_{a}$ since it couples only with the real component of the modulus. The production rate of a vectorial dark matter would have the same qualitative features as the fermionic case but with a steeper bend at high temperatures, since the temperature dependence in the heavy regime is $T^{12}$ in the vector dark matter case and $T^{10}$ in the fermionic case.

The presence of the pole regions depends on the low- and high-temperature thresholds. It will not appear if the Boltzmann suppression takes place before it (as in the green curve, for $x \sim 100$ ). Since the Universe has a maximal temperature (fixed to $10^{12} \mathrm{GeV}$ in Fig. 1), the production rate will have maximal values at $x=10^{-2}, 10$, and $10^{3}$. As a consequence, the pole due to the real component exchange would not contribute for the cases in orange and blue.

\section{B. Relic abundance}

From the approximate rates given in the last section, we can get an idea about the parameter space in agreement with the inferred value of the dark matter relic density $\Omega h^{2}=0.1200 \pm 0.0012$ [1]. Taking the limit of heavy moduli, we find

$$
\frac{\Omega h^{2}}{0.12} \approx \begin{cases}\frac{B_{F}^{(12)}}{35.5} \alpha_{S}^{2} \frac{\alpha_{\mathrm{SM}}^{2}}{5}\left(\frac{m_{\mathrm{DM}}}{1.2 \times 10^{14} \mathrm{GeV}}\right)\left(\frac{T_{\mathrm{RH}}}{10^{10} \mathrm{GeV}}\right)^{7}\left(\frac{\Lambda}{10^{15} \mathrm{GeV}}\right)^{-4}\left(\frac{m_{t}}{10^{13} \mathrm{GeV}}\right)^{-4} & \text { (scalarDM) } \\ \frac{B_{F}^{(10)}}{3.68}\left(\frac{m_{\mathrm{DM}}}{3.2 \times 10^{10} \mathrm{GeV}}\right)^{3}\left(\frac{T_{\mathrm{RH}}}{10^{10} \mathrm{GeV}}\right)^{5}\left(\frac{\Lambda}{10^{15} \mathrm{GeV}}\right)^{-4}\left[\frac{\left(\alpha_{\mathrm{V}}^{\chi}\right)^{2}}{2} \frac{\alpha_{\mathrm{SM}}^{2}}{5}\left(\frac{m_{t}}{10^{13} \mathrm{GeV}}\right)^{-4}+\frac{\left(\beta_{\mathrm{A}}^{\prime}\right)^{2}}{2} \beta_{G}^{2}\left(\frac{m_{a}}{10^{12} \mathrm{GeV}}\right)^{-4}\right] & \text { (fermionic DM), } \\ \frac{B_{F}^{(12)}}{35.5}\left(\frac{m_{\mathrm{DM}}}{1.5 \times 10^{12} \mathrm{GeV}}\right)\left(\frac{T_{\mathrm{RH}}}{10^{10} \mathrm{GeV}}\right)^{7}\left(\frac{\Lambda}{10^{15} \mathrm{GeV}}\right)^{-4}\left[\frac{\alpha_{V}^{2}}{2} \frac{\alpha_{\mathrm{SM}}^{2}}{25}\left(\frac{m_{t}}{10^{13} \mathrm{GeV}}\right)^{-4}+\frac{\beta_{V}^{2}}{2} \beta_{G}^{2}\left(\frac{m_{a}}{10^{12} \mathrm{GeV}}\right)^{-4}\right] & \text { (vectorialDM). }\end{cases}
$$




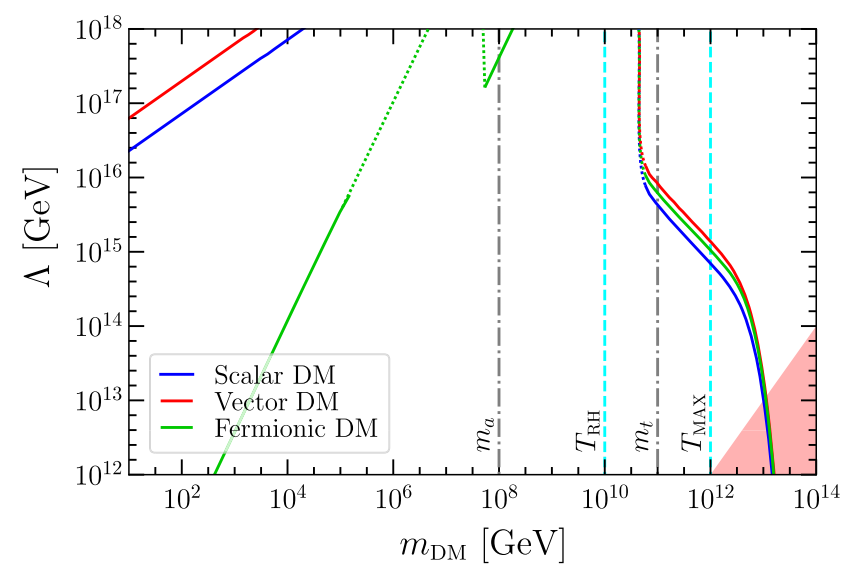

FIG. 2. Contours respecting $\Omega h^{2}=0.12$ in the $\left(m_{\mathrm{DM}}, \Lambda\right)$ plane for the real and imaginary parts of the modulus with masses $m_{t}=$ $10^{11} \mathrm{GeV}$ and $m_{a}=10^{8} \mathrm{GeV}$, respectively. Solid (dotted) curves are for the exact (approximate) computation of the production rates. For illustrative purposes, we set $T_{\mathrm{RH}}=10^{10} \mathrm{GeV}$, $T_{\mathrm{MAX}}=100 T_{\mathrm{RH}}$, and all couplings are set to unity. The region in red is not reliable since $\Lambda<m_{\mathrm{DM}}$.

It is important to emphasize that the expressions in Eq. (25) are computed with a simplified hypothesis, specifically in the limit $m_{t, a} \gg T_{\mathrm{MAX}}$. Comparing Eq. (25) with our numerical results, we notice that pole effects due to the exchange of $t, a$ can be important even when $m_{\mathrm{DM}}$ lies above $T_{\mathrm{MAX}}$ as the enhancement due to a small width can compensate the Boltzmann suppression $e^{-m_{\mathrm{DM}} / T_{\mathrm{MAX}}}$.

Our results are summarized in Figs. 2 and 3, where we plot the contours in the parameter space $\left(m_{\mathrm{DM}}, \Lambda\right)$ corresponding to $\Omega h^{2}=0.12$ for the scalar, fermionic, and vectorial dark matter (blue, green, and red curves, respectively). For simplicity, all of the couplings in the Lagrangian are set to unity. The split between the reheating and maximal temperatures depends, of course, on the inflationary model. We set this split to be $T_{\mathrm{MAX}}=$ $100 T_{\mathrm{RH}}$ (inspired by a Starobinsky-like potential [36]),

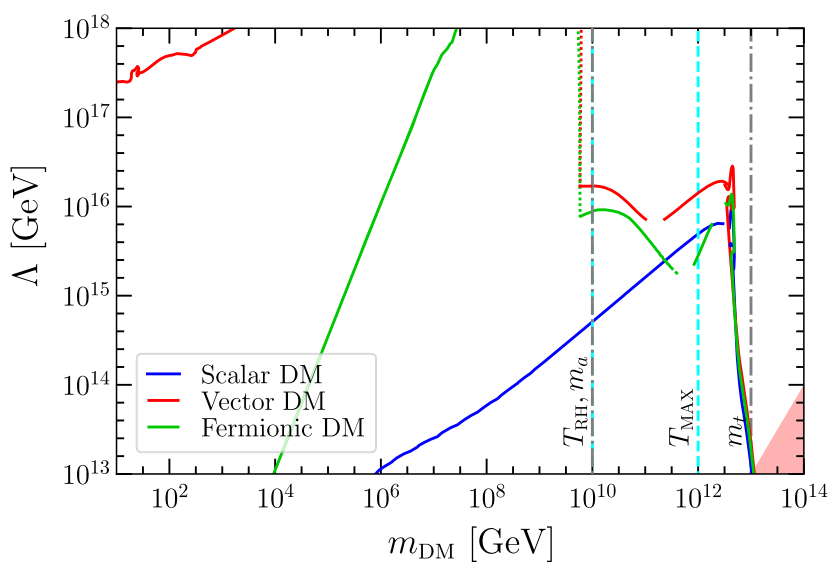

FIG. 3. Contours respecting $\Omega h^{2}=0.12$ in the $\left(m_{\mathrm{DM}}, \Lambda\right)$ plane for same parameter values as in Fig. 2 but for heavier moduli: $m_{a}=10^{10} \mathrm{GeV}$ and $m_{t}=10^{13} \mathrm{GeV}$. which sets the initial condition for the inflaton energy density (after the end of inflation) to be $\rho_{\text {end }} \simeq 0.175 m_{\phi}^{2} M_{P}^{2}$ [37], for an inflaton mass of $m_{\phi}=10^{13} \mathrm{GeV}$ and for an inflaton decay width $\Gamma_{\phi}=\alpha_{\phi} m_{\phi}$ with $\alpha_{\phi}=10^{-8}$. We set the reheating scale to be $10^{10} \mathrm{GeV}$. In Fig. 2 we set $m_{a}=10^{8} \mathrm{GeV}$ and $m_{t}=10^{11} \mathrm{GeV}$, whereas we explore a scenario with heavier mediators in Fig. 3, with $m_{a}=$ $10^{10} \mathrm{GeV}$ and $m_{t}=10^{13} \mathrm{GeV}$.

With this set of parameters, it is imperative to consider the presence of the inflaton energy density in the Hubble rate. Indeed, our complete analysis is performed by solving the complete set of Boltzmann equations, and integrating over the whole phase space with Bose-Einstein distribution functions for the initial states (since standard fermions do not contribute to dark matter production in our scenario). We compute the triple integral of the production rates using a Monte Carlo method (Vegas), as given by the CUBA package [38]. Depending on the parameter choice, the Monte Carlo integration outputs close to the pole regions (and thus the relic density) are highly oscillatory, rendering unreliable results. Because of this, while we are always solving the set of Boltzmann equations, we interpolate the relic density contours found when we compute the rates exactly (solid curves) with the contours found when we use approximate rates (dotted curves). ${ }^{8}$

The first thing the reader might notice in each figure is the stronger dependence of the fermionic contour on the dark matter mass, as compared to the scalar and vectorial cases which have a linear dark matter mass dependence coming from the relic density definition. This is easy to understand since the amplitude for the annihilation of the Standard Model states into fermionic dark matter depend explicitly on the dark matter mass [see Eq. (9)]. It is therefore easier to see in the fermionic contours the following generic feature: the lighter the dark matter, the smaller the new physics scale for the same relic density value. On the other hand, the contours of the scalar and vectorial dark matter have similar behavior, as suggested by the approximate expressions for the rate in Eq. (23). Comparing the scalar and vectorial cases in each figure, we see that for any dark matter mass, the same relic density value is achieved for larger values of $\Lambda$ in the vectorial case, since a vectorial dark matter receives a contribution from the imaginary part of modulus and the rate is therefore higher, which is not the case for the scalar dark matter. This feature is more accentuated in Fig. 3, as we will be able to understand in what follows.

In the parameter region where $m_{t, a}>2 m_{\mathrm{DM}}$, the mediators can decay on shell to the dark matter particles

\footnotetext{
${ }^{8}$ At a given temperature, the production rate due to the exchange of a mediator $j$ is approximated by $R^{j}(T)=$ $e^{-m_{\mathrm{DM}} / T}\left(\min R_{\text {light }}^{j}, R_{\text {heavy }}^{j}\right)$; in this way, we can account for the six different regimes of production as well as for the Boltzmann suppression.
} 
whenever the pole can be reached $\left(m_{t, a}<T_{\mathrm{MAX}}\right)$. The dark matter production in this region of the parameter space is therefore enhanced and we can understand that by increasing $m_{\mathrm{DM}}, \Lambda$ needs to be increased as well to provide the same relic density. So, the contour of $\Omega h^{2}=0.12$ monotonically increases as $m_{\mathrm{DM}}$ is increased until the threshold for on-shell production is reached, for $m_{\mathrm{DM}} \simeq m_{t, a} / 2$. In the parameter region with $m_{\mathrm{DM}}>m_{t, a} / 2$, the dark matter is produced from the Standard Model through off-shell mediators. Thus, in this regime the cross section and the rate are much lower compared to the pole-enhanced region. As the rate is much lower in this regime, the effective scale $(\Lambda)$ also needs to be decreased to much lower values so as to satisfy the Planck constraint on the dark matter relic density. This is why we can observe a very sharp transition between the onshell and off-shell production regimes. This decrease in $\Lambda$ continues as we increase the dark matter mass, and becomes drastically accentuated because of the Boltzmann suppression in the rate, as we saw in Fig. 1. The heavier the dark matter, the lower its production, and then a smaller $\Lambda$ is needed to compensate the loss of production. The process goes on until the point where it becomes impossible to produce dark matter from the Standard Model. In fact, if dark matter is that heavy, our effective theory approach is no longer on a firm footing since we would enter into an unreliable region of our parameter space, with $\Lambda<m_{\mathrm{DM}}$ (red shaded region).

Because of their accentuated dark matter mass dependence, the fermionic dark matter contours allow much lower effective scale values for the entire region where dark matter is produced through the on-shell decay of the mediators. As the dark matter mass increases to $m_{\mathrm{DM}} \gtrsim$ $m_{t, a} / 2$ the off-shell production becomes the dominant one and the rate slowly approaches the rate of the other two dark matter cases. As a result of this, in this regime the allowed contour for fermionic dark matter mimics the scalar and vectorial dark matter curves. Notice that in the fermionic contour we see a kink around $m_{\mathrm{DM}} \simeq m_{a} / 2^{9}$; this is due to the fact that, before this region, the fermionic dark matter production was due to the on-shell decay of both the real and imaginary parts of the moduli (pole region). As the dark matter mass reaches $m_{\mathrm{DM}} \simeq m_{a} / 2$, the dark matter production through the imaginary part of the moduli becomes off shell, while production through the real part of the moduli remains on shell. At this point, the dark matter production rate decreases and as a result there is a dip in the curve to compensate for this change. Above this regime, the slope of the curve changes as the dominant contribution to the rate is only through the onshell exchange of the real part of the moduli.

Finally, we can understand the main difference between Figs. 2 and 3. Heavier mediators lead to suppressed rates,

\footnotetext{
${ }^{9}$ For the vectorial case, this kink would happen for unrealistically high values of $\Lambda$, which are not displayed in our results.
}

which brings the effective scale to lower (and in our case more reasonable) values. From the fermionic and vectorial dark matter contours, we observe in Fig. 3 viable regions which could not be present in Fig. 2, corresponding to the combination of off-shell production from imaginary modulus and on-shell production of real modulus that happens for dark matter masses between $T_{\mathrm{RH}}$ and $T_{\mathrm{MAX}}$. Concerning the scalar contour, we see in Fig. 3 that the enhancement of the on-shell production from the real modulus (the only mediator possible in this case) is not more efficient than the suppression due to the exchange of a very heavy modulus.

We finalize our discussion with the following lesson for the reader. The derivative couplings of the operators connecting the visible and dark sectors, due to moduli field exchanges, generate high temperature dependences in the production rates. As a consequence, the dark matter candidates considered in the present work are mainly produced during the reheating process in the early Universe. Additionally, the interplay of the pole enhancements due to the two moduli mediators controls the regions of the parameter space which can account for the right amount of dark matter in the Universe.

\section{CONCLUSION}

We showed in this work that moduli fields which are present in several extensions of the Standard Model, even if very heavy, can play the role of a mediator between the dark sector and the Standard Model. Through its couplings to the thermal bath, dark matter can be produced at a sufficient rate to fulfill the observed relic abundance. The main production takes place through an ultraviolet freeze-in mechanism where the majority of dark matter is created at the early stage of reheating. Our main results are summarized in Figs. 2 and 3, where the correct relic abundance can be obtained for a broad range of dark matter mass and an effective scale $\Lambda$ around the unification/ string scale. Finally, our results are quite general, and can be applied to any ultraviolet models where moduli fields are present.

\section{ACKNOWLEDGMENTS}

The authors especially want to thank W. Buchmüller and $\mathrm{K}$. Olive for detailed and insightful discussions. This research has been supported by the (Indo-French) CEFIPRA/IFCPAR Project No. 5404-2. Support from CNRS LIA-THEP and the INFRE-HEPNET of CEFIPRA/IFCPAR is also acknowledged. D.C. would like to thank the CERN theory group for their hospitality and support. E. D. was supported in part by the ANR Grant Black-dS-String No. ANR-16-CE31-0004-01. M. D. acknowledges support from the Brazilian PhD program "Ciências sem Fronteiras"-CNPQ Process No. 202055/ 2015-9. This work was also supported by the France-US 
PICS No. 06482, PICS MicroDark. This project has received funding/support from the European Union's Horizon 2020 research and innovation programme under the Marie Sklodowska-Curie: RISE InvisiblesPlus (grant agreement No. 690575) and the ITN Elusives (Grant agreement No. 674896).

\section{APPENDIX A: WIDTH OF THE MODULI FIELDS}

Here we provide the expressions for the decay widths of the moduli fields. ${ }^{10}$ The real components of the moduli may decay into scalars and vectors of the Standard Model, since the decays into fermions are not allowed above the electroweak symmetry breaking scale. Therefore, we have

$$
\begin{aligned}
\Gamma_{t} & =4 \Gamma_{t \rightarrow H H}+12 \Gamma_{t \rightarrow G G}+\Gamma_{t \rightarrow \mathrm{DMDM}} \\
& =\frac{m_{t}^{3}}{\pi \Lambda^{2}}\left(\frac{f_{H}\left(m_{t}^{2}\right)^{2}}{8} \sqrt{1-\frac{4 \mu_{0}^{2}}{m_{t}^{2}}}+\frac{3}{16} \alpha_{G}^{2}\right)+\Gamma_{t \rightarrow \mathrm{DMDM}} .
\end{aligned}
$$

The distinct ways of writing the mass parameter of the Higgs lead us to define the function ${ }^{11}$

$$
f_{H}(x) \equiv \begin{cases}\alpha_{H} & \text { [case of Eq. (3)] } \\ \alpha_{H}\left(1-\frac{2 \mu_{0}^{2}}{x}\right)+\frac{2 \Lambda}{x} \frac{\langle F\rangle}{M_{P}} & \text { [case of Eq. (4)] }\end{cases}
$$

In the case of the imaginary component, decay into scalars is prohibited, and we have simply

$$
\begin{aligned}
\Gamma_{a} & =12 \Gamma_{a \rightarrow G G}+\Gamma_{a \rightarrow \mathrm{DMDM}} \\
& =\frac{m_{a}^{3}}{\pi \Lambda^{2}} 3 \beta_{G}^{2}+\Gamma_{a \rightarrow \mathrm{DMDM}} .
\end{aligned}
$$

The partial decay widths of the real and imaginary parts of the modulus into dark matter read, respectively,

$$
\begin{aligned}
\Gamma_{t \rightarrow \mathrm{DMDM}}= & \frac{m_{t}^{3}}{\pi \Lambda^{2}} \sqrt{1-\frac{4 m_{\mathrm{DM}}^{2}}{m_{t}^{2}}} \\
& \times \begin{cases}\frac{\alpha_{S}^{2}}{32}\left(1-\frac{2 m_{\mathrm{DM}}^{2}}{m_{t}^{2}}\right)^{2} & \text { for } S, \\
\frac{\left(\alpha_{V}^{\chi}\right)^{2}}{8} \frac{m_{\mathrm{DM}}^{2}}{m_{t}^{2}}\left(1-\frac{4 m_{\mathrm{DM}}^{2}}{m_{t}^{2}}\right) & \text { for } \chi, \\
\frac{\beta_{V}^{2}}{64}\left(1-\frac{4 m_{\mathrm{DM}}^{2}}{m_{t}^{2}}+\frac{6 m_{\mathrm{DM}}^{4}}{m_{t}^{4}}\right) & \text { for } \mathcal{V},\end{cases}
\end{aligned}
$$

\footnotetext{
${ }^{10}$ Finite-temperature effects on the decay width of moduli (as discussed, for instance, in Ref. [39]) will not be relevant for us since we will not consider the moduli as ultrarelativistic species in our analysis.

${ }^{11}$ In what follows, we assume that both couplings of moduli to the Higgs are equal, so that $\alpha_{\mu}=\alpha_{H}$.
}

and

$$
\begin{aligned}
\Gamma_{a \rightarrow \mathrm{DMDM}}= & \frac{m_{a}^{3}}{\pi \Lambda^{2}} \sqrt{1-\frac{4 m_{\mathrm{DM}}^{2}}{m_{a}^{2}}} \\
& \times \begin{cases}0 & \text { for } S, \\
\frac{1}{8}\left(\beta_{\chi} \beta_{5}^{\chi}\right)^{2} \frac{m_{\mathrm{DM}}^{2}}{m_{a}^{2}} & \text { for } \chi \\
\frac{\beta_{\mathcal{V}}^{2}}{4}\left(1-\frac{4 m_{\mathrm{DM}}^{2}}{m_{a}^{2}}\right) & \text { for } \mathcal{V} .\end{cases}
\end{aligned}
$$

\section{APPENDIX B: SQUARED AMPLITUDES}

In what follows, we provide the expressions for the squared amplitudes of $s$-channel SM annihilations into DM candidates of spin $s_{f}\left(|\mathcal{M}|_{s_{f}}^{2}\right)$ :

$$
\begin{array}{r}
|\mathcal{M}|_{0}^{2}=\frac{\alpha_{S}^{2}}{\Lambda^{4}} \frac{s^{4}\left(1-\frac{2 m_{\mathrm{DM}}^{2}}{s}\right)^{2}}{\left(s-m_{t}^{2}\right)^{2}+m_{t}^{2} \Gamma_{t}^{2}} \sum_{s_{i}} \lambda_{s_{i}, 0}^{t}(s), \\
|\mathcal{M}|_{1 / 2}^{2}=\frac{\left(\alpha_{\chi}\right)^{2}}{\Lambda^{4}} \frac{m_{\mathrm{DM}}^{2} s^{3}\left(1-\frac{4 m_{\mathrm{DM}}^{2}}{s}\right)}{\left(s-m_{t}^{2}\right)^{2}+m_{t}^{2} \Gamma_{t}^{2}} \sum_{s_{i}} \lambda_{s_{i}, 1 / 2}^{t}(s) \\
+\frac{\left(\beta_{\chi} \beta_{5}^{\chi}\right)^{2}}{\Lambda^{4}} \frac{m_{\mathrm{DM}}^{2} s^{3}}{\left(s-m_{a}^{2}\right)^{2}+m_{a}^{2} \Gamma_{a}^{2}} \sum_{s_{i}} \lambda_{s_{i}, 1 / 2}^{a}(s), \\
|\mathcal{M}|_{1}^{2}=\frac{\alpha_{\mathcal{V}}^{2}}{\Lambda^{4}} \frac{s^{4}\left(1-\frac{4 m_{\mathrm{DM}}^{2}}{s}+\frac{6 m_{\mathrm{DM}}^{4}}{s^{2}}\right)}{\left(s-m_{t}^{2}\right)^{2}+m_{t}^{2} \Gamma_{t}^{2}} \sum_{s_{i}} \lambda_{s_{i}, 1}^{t}(s) \\
+\frac{\beta_{\mathcal{V}}^{2}}{\Lambda^{4}} \frac{s^{4}\left(1-\frac{4 m_{\mathrm{DM}}^{2}}{s}\right)}{\left(s-m_{a}^{2}\right)^{2}+m_{a}^{2} \Gamma_{a}^{2}} \sum_{s_{i}} \lambda_{s_{i}, 1}^{a}(s) .
\end{array}
$$

We parametrize the contribution of the $N_{i}$ SM initial states with spin $s_{i}$ for the production of DM of spin $s_{f}$ through the exchange of a field $j$ by $\lambda_{s_{i}, s_{f}}^{j}$, which may be functions of the Mandelstam variable $s$ and the masses and couplings involved in the processes. They are given in Table I.

TABLE I. Coefficients of the squared amplitudes: $\lambda_{s_{i}, s_{f}}^{t}$ (left) and $\lambda_{s_{i}, s_{f}}^{a}$ (right) [Eqs. (B1)-(B3)].

\begin{tabular}{lccc}
\hline \hline & \multicolumn{3}{c}{ DM } \\
\cline { 2 - 4 } SM & spin-0 & spin-1/2 & spin-1 \\
\hline spin-0 & $f_{H}^{2}(s)$ & $2 f_{H}^{2}(s)$ & $1 / 2 f_{H}^{2}(s)$ \\
spin-1/2 & 0 & 0 & 0 \\
spin-1 & $1 / 2 \alpha_{G}^{2}$ & $\alpha_{G}^{2}$ & $1 / 4 \alpha_{G}^{2}$ \\
\hline \hline & \multicolumn{3}{c}{ DM } \\
\cline { 2 - 4 } SM & spin-0 & spin-1/2 & spin-1 \\
\hline spin-0 & 0 & 0 & 0 \\
spin-1/2 & 0 & 0 & 0 \\
spin-1 & 0 & $16 \beta_{G}^{2}$ & $64 \beta_{G}^{2}$ \\
\hline \hline
\end{tabular}


The total production rate of the dark matter candidate with spin $s_{f}$ from $N_{i}$ ultrarelativistic $\left(m_{i} \ll T, \sqrt{s}\right)$ thermal particles of spin $s_{i}$ in terms of the Mandelstam variable $s$ is given by

$$
\begin{aligned}
R(T)_{s_{f}}= & \sum_{s_{i}} R(T)_{s_{i} \rightarrow s_{f}} \\
= & \frac{4 \pi S_{f}}{2048 \pi^{6}} \int_{4 m_{\mathrm{DM}}^{2}}^{\infty} d s \sqrt{1-\frac{4 m_{\mathrm{DM}}^{2}}{s}} \\
& \times\left(\sum_{s_{i}} N_{i} S_{i}|\mathcal{M}|_{s_{i} \rightarrow s_{f}}^{2}\right) \int_{0}^{\infty} d p_{1}^{i} f_{1}^{i} \int_{\frac{s}{4 p_{i_{1}}}}^{\infty} d p_{2}^{i} f_{2}^{i},
\end{aligned}
$$

where the symmetrization factors $S_{i, f}=1 / n_{i, f}$ ! account for $n_{i, f}$ identical particles in the initial/final state and $f_{1,2}^{i}=$ $\left(e^{-p_{1,2}^{i} / T} \pm 1\right)^{-1}$ are the distribution functions of the initialstate particles.

The integration over the initial momenta in Eq. (B4) is approximately given by $T \sqrt{s} K_{1}\left(\frac{\sqrt{s}}{T}\right)$, which means that after integrating over $s$ we recognize the light, pole, and heavy regimes of the mediator by comparing the temperature of the thermal bath with the mediator mass. In
TABLE II. Coefficients appearing in the approximate rates [Eqs. (23) and (24)]: $\delta_{s_{f}}^{t}$ (left) and $\delta_{s_{f}}^{a}$ (right). We have defined $\alpha_{\text {SM }}^{2} \equiv 2 \alpha_{H}^{2}+3 \alpha_{G}^{2}$, since we assume $\mu_{0}^{2} \ll s$. Except for the NWA cases, we have used Bose-Einstein statistics for the initial-state distribution functions.

\begin{tabular}{lccc}
\hline \hline & \multicolumn{3}{c}{$\mathrm{DM}$} \\
regimes of $t$ & $\operatorname{spin}-0$ & $\operatorname{spin}-1 / 2$ & spin-1 \\
\cline { 2 - 4 } Light & $\frac{\pi^{3}}{108000} \alpha_{S}^{2} \alpha_{\mathrm{SM}}^{2}$ & $\frac{\zeta(3)^{2}}{8 \pi^{5}}\left(\alpha_{V}^{\chi}\right)^{2} \alpha_{\mathrm{SM}}^{2}$ & $\frac{\pi^{3}}{21600} \alpha_{\mathcal{V}}^{2} \alpha_{\mathrm{SM}}^{2}$ \\
NWA & $\frac{1}{1024 \pi^{4}} \alpha_{S}^{2} \alpha_{\mathrm{SM}}^{2}$ & $\frac{1}{256 \pi^{4}}\left(\alpha_{V}^{\chi}\right)^{2} \alpha_{\mathrm{SM}}^{2}$ & $\frac{1}{2048 \pi^{4}} \alpha_{\mathcal{V}}^{2} \alpha_{\mathrm{SM}}^{2}$ \\
Heavy & $\frac{64 \pi^{7}}{19845} \alpha_{S}^{2} \alpha_{\mathrm{SM}}^{2}$ & $\frac{72 \zeta(5)^{2}}{\pi^{5}}\left(\alpha_{V}^{\chi}\right)^{2} \alpha_{\mathrm{SM}}^{2}$ & $\frac{32 \pi^{7}}{19845} \alpha_{\mathcal{V}}^{2} \alpha_{\mathrm{SM}}^{2}$ \\
\hline \hline & \multicolumn{3}{c}{$\mathrm{DM}$} \\
regimes of $a$ & $\operatorname{spin}-0$ & $\operatorname{spin}-1 / 2$ & $\operatorname{spin}-1$ \\
\hline Light & 0 & $\frac{6 \zeta(3)^{2}}{\pi^{5}}\left(\beta_{A}^{\chi}\right)^{2} \beta_{G}^{2}$ & $\frac{8 \pi^{3}}{225} \beta_{\mathcal{V}}^{2} \beta_{G}^{2}$ \\
NWA & 0 & $\frac{3}{16 \pi^{4}}\left(\beta_{A}^{\chi}\right)^{2} \beta_{G}^{2}$ & $\frac{3}{8 \pi^{4}} \beta_{\mathcal{V}}^{2} \beta_{G}^{2}$ \\
Heavy & 0 & $\frac{3456 \zeta(5)^{2}}{\pi^{5}}\left(\beta_{A}^{\chi}\right)^{2} \beta_{G}^{2}$ & $\frac{8192 \pi^{7}}{6615} \beta_{\mathcal{V}}^{2} \beta_{G}^{2}$ \\
\hline \hline
\end{tabular}

Table II we provide the proportionality constants of the approximate expressions for the rates given in Eqs. (23) and (24).
[1] Planck Collaboration, arXiv:1807.06209.

[2] E. Aprile et al. (XENON100 Collaboration), Phys. Rev. Lett. 109, 181301 (2012).

[3] D. S. Akerib et al. (LUX Collaboration), Phys. Rev. Lett. 118, 021303 (2017).

[4] C. Fu et al. (PANDAX-II Collaboration), Phys. Rev. Lett. 118, 071301 (2017).

[5] J. A. Casas, D. G. Cerdeño, J. M. Moreno, and J. Quilis, J. High Energy Phys. 17 (2017) 36; A. Djouadi, O. Lebedev, Y. Mambrini, and J. Quevillon, Phys. Lett. B 709, 65 (2012); A. Djouadi, A. Falkowski, Y. Mambrini, and J. Quevillon, Eur. Phys. J. C 73, 2455 (2013); O. Lebedev, H. M. Lee, and Y. Mambrini, Phys. Lett. B 707, 570 (2012); Y. Mambrini, Phys. Rev. D 84, 115017 (2011).

[6] J. Ellis, A. Fowlie, L. Marzola, and M. Raidal, Phys. Rev. D 97, 115014 (2018); G. Arcadi, Y. Mambrini, and F. Richard, J. Cosmol. Astropart. Phys. 15 (2015) 018; J. Kearney, N. Orlofsky, and A. Pierce, Phys. Rev. D 95, 035020 (2017); M. Escudero, A. Berlin, D. Hooper, and M.-X. Lin, J. Cosmol. Astropart. Phys. 16 (2016) 29.

[7] A. Alves, S. Profumo, and F. S. Queiroz, J. High Energy Phys. 14 (2014) 63; O. Lebedev and Y. Mambrini, Phys. Lett. B 734, 350 (2014); G. Arcadi, Y. Mambrini, M. H. G. Tytgat, and B. Zaldivar, J. High Energy Phys. 14 (2014) 134.
[8] G. Arcadi, M. Dutra, P. Ghosh, M. Lindner, Y. Mambrini, M. Pierre, S. Profumo, and F. S. Queiroz, Eur. Phys. J. C 78, 203 (2018).

[9] L. J. Hall, K. Jedamzik, J. March-Russell, and S. M. West, J. High Energy Phys. 10 (2010) 80; 1X. Chu, T. Hambye, and M. H. G. Tytgat, J. Cosmol. Astropart. Phys. 12 (2012) 034; X. Chu, Y. Mambrini, J. Quevillon, and B. Zaldivar, J. Cosmol. Astropart. Phys. 14 (2014) 034.

[10] N. Bernal, M. Heikinheimo, T. Tenkanen, K. Tuominen, and V. Vaskonen, Int. J. Mod. Phys. A 32, 1730023 (2017).

[11] Y. Mambrini, K. A. Olive, J. Quevillon, and B. Zaldivar, Phys. Rev. Lett. 110, 241306 (2013); Y. Mambrini, N. Nagata, K. A. Olive, J. Quevillon, and J. Zheng, Phys. Rev. D 91, 095010 (2015); Y. Mambrini, N. Nagata, K. A. Olive, and J. Zheng, Phys. Rev. D 93, 111703(R) (2016).

[12] G. Bhattacharyya, M. Dutra, Y. Mambrini, and M. Pierre, Phys. Rev. D 98, 035038 (2018).

[13] N. Bernal, M. Dutra, Y. Mambrini, K. A. Olive, M. Peloso, and M. Pierre, Phys. Rev. D 97, 115020 (2018).

[14] K. Benakli, Y. Chen, E. Dudas, and Y. Mambrini, Phys. Rev. D 95, 095002 (2017); E. Dudas, Y. Mambrini, and K. Olive, Phys. Rev. Lett. 119, 051801 (2017); E. Dudas, T. Gherghetta, Y. Mambrini, and K. A. Olive, Phys. Rev. D 96, 115032 (2017); E. Dudas, T. Gherghetta, K. Kaneta, Y. Mambrini, and K. A. Olive, Phys. Rev. D 98, 015030 (2018). 
[15] M. A. G. Garcia, Y. Mambrini, K. A. Olive, and M. Peloso, Phys. Rev. D 96, 103510 (2017).

[16] M. A. G. Garcia and M. A. Amin, Phys. Rev. D 98, 103504 (2018).

[17] T. R. Taylor and G. Veneziano, Phys. Lett. B 213, 450 (1988).

[18] T. Damour and A. M. Polyakov, Nucl. Phys. B423, 532 (1994).

[19] P. Binetruy and E. Dudas, Phys. Lett. B 338, 23 (1994); Nucl. Phys. B442, 21 (1995); B451, 31 (1995).

[20] C. Kounnas, I. Pavel, and F. Zwirner, Phys. Lett. B 335, 403 (1994).

[21] S. Dimopoulos and G. F. Giudice, Phys. Lett. B 379, 105 (1996).

[22] I. Antoniadis, S. Dimopoulos, and G. Dvali, Nucl. Phys. B516, 70 (1998).

[23] F. Feruglio, in From My Vast Repertoire...: Guido Altarelli's Legacy, edited by A. Levy, S. Forte, and G. Ridolfi (World Scientific, Singapore, 2019), pp. 227-266; J. C. Criado and F. Feruglio, SciPost Phys. 5, 042 (2018).

[24] A. Kusenko, M. Loewenstein, and T. T. Yanagida, Phys. Rev. D 87, 043508 (2013).

[25] P. S. B. Dev, A. Mazumdar, and S. Qutub, Front. Phys. 2, 26 (2014).

[26] R. Allahverdi, B. Dutta, R. N. Mohapatra, and K. Sinha, Phys. Rev. Lett. 111, 051302 (2013).

[27] B. S. Acharya, G. Kane, S. Watson, and P. Kumar, Phys. Rev. D 80, 083529 (2009).
[28] B. S. Acharya, P. Kumar, K. Bobkov, G. Kane, J. Shao, and S. Watson, J. High Energy Phys. 06 (2008) 064.

[29] T. Moroi and L. Randall, Nucl. Phys. B570, 455 (2000).

[30] S.-M. Choi, Y. Hochberg, E. Kuflik, H. M. Lee, Y. Mambrini, H. Murayama, and M. Pierre, J. High Energy Phys. 10 (2017) 162.

[31] G. D. Coughlan, W. Fischler, E. W. Kolb, S. Raby, and G. G. Ross, Phys. Lett. 131B, 59 (1983); T. Banks, D. B. Kaplan, and A. E. Nelson, Phys. Rev. D 49, 779 (1994); B. de Carlos, J. A. Casas, F. Quevedo, and E. Roulet, Phys. Lett. B 318, 447 (1993); P. Binetruy, in Particle Physics and Cosmology: The Interface. Proceedings, NATO Advanced Study Institute, School, Cargese, France, 2003 (Springer, Dordrecht, 2003), pp. 181-234.

[32] M. Kawasaki, K. Kohri, and N. Sugiyama, Phys. Rev. Lett. 82, 4168 (1999); Phys. Rev. D 62, 023506 (2000); S. Hannestad, Phys. Rev. D 70, 043506 (2004).

[33] G. F. Giudice, E. W. Kolb, and A. Riotto, Phys. Rev. D 64, 023508 (2001).

[34] A. Mazumdar and B. Zaldávar, Nucl. Phys. B886, 312 (2014).

[35] T. Hahn, Comput. Phys. Commun. 168, 78 (2005).

[36] A. A. Starobinsky, Phys. Lett. 91B, 99 (1980).

[37] J. Ellis, M. A. G. Garcia, D. V. Nanopoulos, K. A. Olive, and M. Peloso, J. Cosmol. Astropart. Phys. 03 (2016) 008.

[38] T. Hahn, Comput. Phys. Commun. 168, 78 (2005).

[39] D. Bodeker, J. Cosmol. Astropart. Phys. 06 (2006) 027. 